Meta

Journal des traducteurs

Translators' Journal

\title{
Analyse de la notion de non-standard dans les dictionnaires bilingues français-anglais
}

\section{Aurélia Paulin}

Volume 42, numéro 1, mars 1997

Lexicologie et terminologie

URI : https://id.erudit.org/iderudit/003803ar

DOI : https://doi.org/10.7202/003803ar

Aller au sommaire du numéro

Éditeur(s)

Les Presses de l'Université de Montréal

ISSN

0026-0452 (imprimé)

1492-1421 (numérique)

Découvrir la revue

Citer cet article

Paulin, A. (1997). Analyse de la notion de non-standard dans les dictionnaires bilingues français-anglais. Meta, 42(1), 55-67. https://doi.org/10.7202/003803ar
Résumé de l'article

L'auteur analyse des dictionnaires bilingues afin d'étudier le problème de l'étiquetage des différents niveaux de langue non standard et propose des solutions pour les lexicographes. Elle affirme qu'il est nécessaire d'analyser la manière dont les lexicographes appréhendent la notion de non standard, les définitions qu'ils en donnent et les critères sur lesquels ils se basent pour conférer à un mot adresse telle ou telle étiquette. Elle étudie ensuite l'évolution de l'étiquetage d'une édition à l'autre pour voir si cette évolution reflète celle de la langue. Enfin, elle (re)définit les niveaux de langue non standard et suggère quelques propositions pour tenter d'améliorer la situation.
Ce document est protégé par la loi sur le droit d'auteur. L'utilisation des services d'Érudit (y compris la reproduction) est assujettie à sa politique d'utilisation que vous pouvez consulter en ligne.

https://apropos.erudit.org/fr/usagers/politique-dutilisation/ 


\title{
ANALYSE DE LA NOTION DE NON-STANDARD DANS LES DICTIONNAIRES BILINGUES FRANÇAIS-ANGLAIS*
}

AURÉlia PAULIN

Université de Franche-Comté, Besançon, France

\begin{abstract}
Résumé
L'auteur analyse des dictionnaires bilingues afin d'étudier le problème de l'étiquetage des différents niveaux de langue non standard et propose des solutions pour les lexicographes. Elle affirme qu'il est nécessaire d'analyser la manière dont les lexicographes appréhendent la notion de non standard, les définitions qu'ils en donnent et les critères sur lesquels ils se basent pour conférer à un mot adresse telle ou telle étiquette. Elle étudie ensuite l'évolution de l'étiquetage d'une édition à l'autre pour voir si cette évolution reflète celle de la langue. Enfin, elle (re)définit les niveaux de langue non standard et suggère quelques propositions pour tenter d" améliorer la situation.
\end{abstract}

\begin{abstract}
The article deals with the way lexicographers label the different registers corresponding to what is generally referred to as substandard (colloquial, slang, vulgar, very vulgar...) and makes suggestions for improvement of this process which she finds highly unsatisfactory. Based on an analysis of the labels used in several general and specialized bilingual French! English dictionaries, the author looks at the evolution of labelling over several editions in an attempt to see whether the process reflects the evolution of both languages. The author feels that in this era of electronic dictionaries, this type of study is more than relevant.
\end{abstract}

En tant que lexicologue dont les recherches ont jusqu'à aujourd'hui porté sur le lexique anglais contemporain non standard, $\mathrm{j}$ 'ai pu appréhender le problème que constitue le traitement dictionnairique de cette langue qui, si l'on utilise une échelle graduée des différents niveaux de langue, se situe «en aval» de la langue standard. La situation est, en effet, loin d'être satisfaisante: la consultation de nombreux ouvrages révèle un flottement important dans les niveaux de langue attribués aux mots adresse et à leurs différents emplois. Cette situation n'est pas sans poser problème au lexicologue pour qui le dictionnaire peut constituer la matière première de son travail, sans parler, plus généralement, des difficultés et des situations quelque peu embarrassantes que peuvent rencontrer l'étudiant anglophone ou francophone, qui, pratiquant la langue étrangère, tombent dans le piège tendu par les différents niveaux de langue. Cela est d'autant plus vrai pour les dictionnaires bilingues, véritables objets de référence des étudiants, qui se réfugient derrière l'autorité dont les premiers sont investis: la réponse recherchée est considérée comme inattaquable ! C'est la raison pour laquelle l'analyse qui suit a pour objet les dictionnaires bilingues généraux les plus utilisés dans les universités françaises, soit le Robert \& Collins, le Harrap's Shorter et enfin le dictionnaire Hachette-Oxford. Elle sera complétée par l'étude d'un dictionnaire bilingue spécialisé : le Harrap's Slang Dictionary, qui servira d'élément de comparaison dans l'analyse du traitement de la notion de non standard qui en est faite. 
Les niveaux de langue, familier, populaire et vulgaire, voire très vulgaire, sont traditionnellement réunis sous le nom de substandard language ou langue non standard. Il est évident qu'il n'y a pas de frontière objective entre ces trois niveaux, pas plus qu'entre le standard et le non-standard. Cet article ne prétend pas révolutionner cet état de fait en transformant ce qui est par définition subjectif en objectif, mais propose, dans un premier temps, une vue panoramique du «paysage dictionnairique» sous la forme d'un état des lieux, pour ensuite examiner dans quelle mesure l'évolution d'une édition à l'autre reflète le renouvellement rapide et permanent propre à cette langue. Cela permettra de suggérer quelques propositions en vue d'une amélioration de la situation. Même si l'accent est mis sur la partie français-anglais des dictionnaires, l'autre partie n'est pas pour autant négligée; les exemples sélectionnés apparaissent en italique, précédés respectivement des abréviations «fra» et «ang» afin d'éviter les ambiguités, et sont suivis de leur(s) sens.

\section{1. ÉTAT DES LIEUX}

\subsection{DICTIONNAIRES GÉNÉRAUX}

\section{I.I. Robert \& Collins, 1993}

Dans l'introduction du Robert \& Collins, édition 1993, les auteurs insistent sur leur volonté de «présenter une image fidèle de la langue telle qu'elle est pratiquée quotidiennement, lue dans les journaux et les revues, parlée en société, entendue dans la rue» ${ }^{\text {. }}$ Cette remarque laisse ainsi présager qu'une place notoire sera réservée à la langue non standard, probablement dans ce qu'elle a de moins vulgaire. De plus, un grand nombre de variétés géographiques sont prétendues être prises en compte, élargissant de ce fait l'horizon. Soulignant le fait que les niveaux de langue constituent des pièges, les auteurs spécifient qu'un système d'indications très précis a été développé sous la forme d'astérisques allant de un à trois, avertissant le lecteur des différents degrés existants. L'échelle va ainsi du «légèrement familier» au «très vulgaire» sans que le soin ne soit pris de les définir. Il faut alors se reporter aux notes d'utilisation ${ }^{2}$ pour en savoir davantage:

«* marque la majeure partie des expressions familières et les incorrections de langage employées dans la langue de tous les jours. Ce signe conseille au lecteur d'être prudent.

** marque les expressions très familières qui sont à employer avec la plus grande prudence par le lecteur étranger, qui devra posséder une grande maîtrise de la langue et savoir dans quel contexte elles peuvent être utilisées.

*** marque le petit nombre d'expressions courantes que le lecteur étranger doit pouvoir reconnaître, mais dont l'emploi risque d'être ressenti comme fortement indécent ou injurieux.»

Il s'agit en fait d'avertissements et non de critères de sélection. La liste d'abréviations ajoute «arg» pour argot et «sl» pour slang au code qui vient d'être mentionné, sans que le sens dans lequel ils sont pris soit clairement énoncé.

Certaines remarques méritent d'être faites concernant le traitement des lexies non standard. Certains mots adresse sont notés ** (très familier) alors qu'ils mériteraient * (familier), tant ils sont passés dans 1'usage et ont ainsi perdu leur caractère choquant ou diffamatoire; c'est le cas de fra. dondon ${ }^{* * *}$ : «big ou fat woman ou girl», fra. gratte**: «guitar», ang. leather**: (US) «portefeuille», ou de ang. iffy**: «aléatoire, problématique». Le problème peut être contraire, c'est-à-dire qu'un mot ou une expression n'est pas notée* ou ** alors qu'il/elle mériterait de l'être; par exemple: fra. tomber sur le dos de quelqu'un: «to drop in on somebody». Assez fréquemment, la mention archaique n'apparaît pas, alors que le mot n'est plus usité dans ce sens : fra. lourde** : «door», fra. 
loupage ${ }^{*}$ : «missing, messing up ${ }^{*} »$, fra. gourance ${ }^{*}$ : «cock-up ${ }^{* *}$, et inversement, fra. maboul(e) est noté + alors qu'il est en usage. Malheureusement, un certain nombre de néologismes apparus dans la langue depuis quelques années sont absents, peuvent être cités: ang. tag: «un graffiti censé imiter une signature»; ang. wrinklie: «une personne âgée»; fra. graf: «abréviation de graffiti»; fra. rappeur: «personne qui danse ou qui joue le $\operatorname{rap} » . .$.

Il faut cependant remarquer que, contrairement à certains dictionnaires, les équivalents donnés dans l'autre langue sont étiquetés du registre qui convient, et que très souvent, lorsque cela est rendu possible par les ressources de la langue, l'équivalent standard coexiste avec l'équivalent non standard. Par conséquent, les risques d'erreurs stylistiques sont réduits et le choix offert au lecteur élargi.

En conclusion, le Robert \& Collins présente des inconvénients qui semblent pour la plupart inhérents à tout dictionnaire qui prend en compte la langue non standard, mais traite ce lexique de manière honorable, ne s'agissant pas d'un dictionnaire spécialisé.

\subsubsection{Harrap's Shorter}

Dans la préface du Harrap's Shorter, seconde édition (1991) révisée en 1993, les auteurs insistent sur le besoin de modernisation par le biais d'une réorganisation, d'une correction et d'un complément d'information dont le but est de refléter l'évolution des deux langues. Il est également annoncé que l'anglais d'Amérique et le français du Canada occupent une place plus importante. La prise en compte de la langue non standard n'est pas mentionnée dans la préface, il faut par conséquent se reporter à la liste des abréviations pour trouver Arg: argot/slang; F : familier/familiar, colloquial ; sl : argot/slang et $\mathrm{V}$ : vulgaire/vulgar, les différents registres, au nombre de trois puisque argot est réservé au français et slang à l'anglais. Au lecteur de deviner l'acception qui est donnée à chacun d'eux, et plus spécifiquement à argot et à slang.

La consultation des nombreuses pages du dictionnaire fait apparaître que argot et slang correspondent à ce qui est noté «populaire/very informal» dans la plupart des dictionnaires bilingues. Des exemples tels que : fra. flouse/ze: «bread, cash»; fra. foireux: «failed»; ang. kip : «roupiller» en sont la preuve. Cet étiquetage n'est pas sans poser des problèmes puisque des mots tels que fra. biffe: «infantry» ou fra. flottard: «naval cadet», sont aussi notés comme argotiques alors qu'ils appartiennent à un groupe social et ne peuvent être traités de la même manière que fra. unc flop(p)ée de: «loads, masses of», ou fra. pétard: «bum», accompagnés pourtant de la même étiquette. Très souvent, les registres conférés à chaque mot ou expression ne semblent pas appropriés; ainsi, fra. se bidonner: «to laugh one's head off»; fra. bidule: «thing, thingummy», notés Arg, ou ang. savvy: «jugeote»; ang. scamp : «fripouille», notés sl, auraient mérité l'étiquette F pour familier. L'évolution de la langue ne semble pas non plus fidèlement reflétée lorsqu'un mot qui n'est plus utilisé n'est pas considéré comme archaïque; c'est le cas de fra. moujingue: «kid, brat»; ou qu'un article est incomplet par l'omission d'un sens non standard pourtant répandu: si fra. morue signifie «tart», il a aussi le sens de: «chick, bird», absent dans le dictionnaire. Plus grave encore est le fait que des expressions ou mots non standard utilisés depuis des années n'aient toujours pas fait leur entrée dans le dictionnaire; référence est faite entre autres à fra. tare: «idiot»; fra. jongler : «to suffer»; ang. rehab: «réhabilitation»; ang. ace: «super, fantastique». Certains mots ou expressions sont considérés comme standard alors que leur emploi peut générer une erreur stylistique; en effet, fra. il a les nerfs en pelote: «his nerves are on edge», ou ang. Pres: «Président» sont davantage familiers. De manière plus générale, il faut déplorer un certain manque de rigueur concernant les équivalents donnés aux mots adresse. Dans certains cas, il n'est pas fait mention du registre auquel ils appartiennent: fra. flanquer $\mathrm{F}$ : «to throw, to pitch, to chuck»; 
ang. crap sl: «camelote, saloperie»; dans d'autres, l'étiquette qui convient est donnée: fra. le travail au noir $\mathrm{F}$ : F «moonlighting», s1 «work(ing) on the black»; ang. crazy: «fou, folle (à lier)»; F «toqué». Quelle raison justifie une telle différence préjudiciable à l'utilisateur?

Ces remarques mériteraient d'être prises en compte afin de limiter les fluctuations et les inadéquations qui peuvent, pour la plupart, induire l'utilisateur en erreur.

\subsubsection{Hachette Oxford, 1994}

Nouveau venu dans le paysage lexicographique bilingue, le dictionnaire HachetteOxford, paru en 1994, présente plusieurs particularités qui peuvent être résumées en ces mots: transparence, innovation et rigueur. La transparence vient du fait que les auteurs précisent de quelle manière ils ont travaillé et recueilli leurs informations. L'innovation se traduit par l'utilisation d'un corpus électronique composé de deux bases de données d'anglais et de français, d'un volume imposant, qui est à l'origine d'une grande rigueur puisque les traductions viennent de la langue réelle et qu'elles sont employées en contexte. Cependant, la langue non standard ne semble pas occuper une place de choix: il est seulement dit que «le dictionnaire recense de nombreux mots nouveaux, des expressions

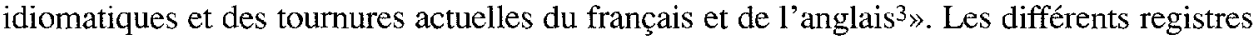
pris en compte apparaissent dans la partie intitulée «Structure du texte» sous une forme innovatrice de notation : o pour familier/informal, o pour populaire/very informal et $\bullet$ pour vulgaire/vulgar or taboo, système qui présente l'avantage d'être facilement repérable dans le texte. Les étiquettes courantes telles que argot et slang ne sont pas mentionnées dans la liste d'abréviations.

Il est essentiel d'analyser maintenant les articles du dictionnaire afin de voir dans quelle mesure l'utilisation d'un corpus électronique a une influence positive sur le traitement des lexies non standard. Assez souvent, et c'est là un aspect positif, le mot adresse non standard est accompagné de plusieurs équivalents dont au moins un standard et un autre non standard. Ainsi fra. asperge o est traduit par: «beanpole o» et par : «stringbean», fra. cossard(e) par: «lazy» et «bone idle o». La même remarque peut être faite mais plus rarement pour les mots adresse standard : fra. exhibitionniste : «exhibitionist, flasher o». II arrive cependant qu'un mot adresse non standard soit traduit par un équivalent standard alors que la langue offre une possibilité appartenant au même registre; c'est le cas de fra. concherie , qui est traduit par: «casual sexual encounter» alors que le mot «screw» existe avec le même sens. Quelquefois, un article peut être incomplet: fra. costume trois pièces ne donne que le sens standard: «suit», alors qu'il signifie aussi: «family jewels». Il arrive qu'un mot rarement usité de nos jours ne soit pas considéré comme un archaïsme, à l'exemple de: fra. as de pique o: «(croupion) parson's nose» ou de fra. couenne $\mathbf{0}$

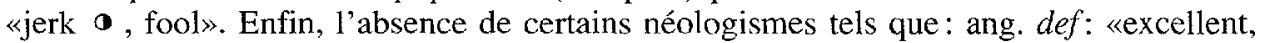
génial»4; ang. clock: «repérer quelqu'un»; fra. bans: «car» ou fra. drôle: «kid» est regrettable.

En conclusion, les inexactitudes et les approximations semblent réduites par l'utilisation du corpus électronique, mais cet outil ne paraît pas à même de régler de manière satisfaisante ni le problème posé par le vieillissement de certaines lexies, ni le passage d'un mot d'un registre à un autre.

\subsection{DICTIONNAIRE SPÉCIALISÉ}

\subsection{Harrap's Slang Dictionary, 1993}

Le choix du Harrap's Slang Dictionary comme élément de comparaison est motivé par plusieurs raisons. Il s'agit d'un des rares dictionnaires bilingues spécialisés qui compte parmi les plus distribués en France, issu du même éditeur que l'un des dictionnaires 
généraux analysés ci-dessus, qui en est à sa deuxième édition. Ces différents critères justifient pleinement sa présence.

Les auteurs de la préface du Harrap's Slang Dictionary soulignent tout d'abord l'évolution rapide de «l'argot et la langue familière», illustrant leur commentaire d'exemples qui montrent le passage de certains mots dans l'oubli et l'apparition de certains autres pour les remplacer: «ce qui était super ou groovy est devenu wicked et crucial; ce qui était sensass ou dans le vent est devenu géant et branchés», et insistent sur le fait que la «langue familière» est sujette, entre autres, aux différentes modes et à l'évolution des tabous. Ils mentionnent par la suite les diverses sources auxquelles ils ont emprunté des mots et expressions dans le but de faire découvrir «la langue verte» à ceux qui s'y intéressent. Sont intégrés les mots «les plus à la mode», maintenus certains termes vieillis, et supprimés des articles certains mots et expressions archaiques qui ont été transférés dans la liste alphabétique de synonymes.

Ce résumé rapide dans lequel sont volontairement cités les expressions et mots utilisés pour faire référence au non-standard appelle un certain nombre de remarques. Le non-standard y est désigné par des termes aussi variés que : argot, langue familière, langue verte, mots et expressions les plus à la mode, termes qui semblent substituables les uns aux autres pour signifier la même réalité, celle du slang, comme l'indique le titre du dictionnaire. À aucun moment les auteurs n'ont jugé nécessaire de définir, d'une part, ce qu'ils entendent par slang, et, d'autre part, les différents registres qui selon eux composent ce dernier. De ce fait, les critères sur lesquels les lexicographes se sont basés pour conférer à tel mot ou à tel sens tel ou tel registre sont passés sous silence. Rien ou presque n'est dit des sources géographiques dans lesquelles les auteurs ont puisé pour établir leur corpus, il faut en effet se contenter «des quatre coins du monde anglophone ou francophone»! Ainsi, le lecteur est laissé dans l'ignorance la plus totale de ce qu'il va trouver dans l'ouvrage, à moins qu'il ne consulte la liste des abréviations dans l'espoir d'obtenir les informations qui lui manquent.

Les différents registres sont au nombre de cinq, matérialisés par: F pour : familier/ familiar, colloquial; $\mathrm{P}$ pour: populaire, argotique/popular, slang; SI pour: expression argotique/slang; $\mathrm{V}$ pour : trivial/vulgar; et VV pour: obscène, ordurier/very vulgar, taboo. À première vue, cette classification paraît logique et sensée, si l'on excepte les deux abréviations $\mathrm{P}$ et Sl. Dans quelle mesure l'étiquette «populaire, argotique» diffère-t-elle de celle d' «expression argotique»? Faut-il en déduire que $P$ s'applique aux lexies simples populaires et argotiques et Sl aux lexies complexes argotiques et non populaires? Une consultation rapide du dictionnaire prouve que cela n'est pas le cas. Faut-il alors penser, assez logiquement d'ailleurs, que P est utilisé dans la partie français-anglais et Sl dans la partie anglais-français? Après vérification, ceci semble être le premier élément de l'énigme. SI apparaît en effet dans la partie anglais-français, accompagné dans la plupart des cas du domaine dans lequel le mot est utilisé ; par exemple : ang. coat $\mathrm{P}$ (police slang) «suspect, -ecte». Les choses semblent plus compliquées pour $\mathrm{P}$, puisqu'il apparaît dans la partie français-anglais, comme le prouve 1'exemple suivant: fra. surbiner $\mathrm{P}$ (surt Police) «to keep (s.o.) under surveillance», mais également dans l'autre partie, comme on l'a vu avec ang. coat. Pourquoi les auteurs ont-ils assimilé deux notions bien différentes (argotique, populaire/slang, popular) sous la même abréviation, alors qu'ils ont ajouté l'étiquette Sl (slang/expression argotique) qu'ils appliquent seulement aux mots anglais? La solution ne réside-t-elle pas dans l'ajout de l'abréviation «arg» pour argot en l'appliquant aux mots français, $P$ conservant seulement le sens de populaire/popular applicables aux deux parties du dictionnaire?

Les différents problèmes qui viennent d'être évoqués ne sont pas sans incidence sur le corpus même du dictionnaire. Certains mots ou expressions sont accompagnés de 
l'abréviation $\mathrm{P}$, alors qu'ils sont maintenant considérés comme familiers, à l'exemple de fra. frichti, nénette, palpitant ou flop(p)ée. D'autres ne méritent plus la mention $\mathrm{F}$ faisant partie intégrante de la langue standard: fra. bricoler, cancre, gagneur, faire l'école buissonnière ou faire des cachotteries. Des exemples tels que fra. Pantruche: «Paris»; fra. bousculer le pot aux fleurs : «exagérer»; fra. entonnoir à musique: «oreille»; fra. frotteuse: «allumette», non accompagnés de la mention vieilli, prouvent que l'évolution de la langue n'a pas été prise en compte.

D'autres remarques d'ordre plus général viennent se greffer aux précédentes. $\mathrm{La}$ première est d'importance, puisque les équivalents sémantiques anglais donnés pour chaque mot adresse ne sont pas accompagnés du registre auquel ils appartiennent. Ainsi, fra. gonzesse, par exemple, est donné comme l'équivalent de: «woman/girl/bird/chick et lady»; fra. pif comme: «nose/conk ou hooter»; cul comme: «backside/buttocks/ bottom/bum/butt et arse», alors qu'ils ne sont pas à considérer de la même manière. Afin d'éviter des erreurs stylistiques préjudiciables, chaque équivalent mériterait donc d'être accompagné de l'abréviation qui convient. Dans le même ordre d'idée, un seul équivalent standard est donné à un mot adresse alors qu'il existe des équivalents nonstandard: c'est le cas entre autres de fra. porte-pipe: «mouth» et de fra. portugaises: «ears», alors qu'il aurait été judicieux de leur adjoindre respectivement: «gob, kisser, cake-hole» et «ear-holes, flappers, jugs» sans que la liste soit pour autant exhaustive. Enfin, certains articles semblent incomplets, à l'image de fra. filochard qui apparaît seulement en tant qu'adjectif signifiant: «resourceful/smart/canny» alors qu'il est utilisé également en tant que nom dans le sens de : «a very thin and tall boy or man».

Ce qui vient d'être dit fait état des lacunes les plus importantes qui, pour la plupart, mériteraient d'être comblées afin de paraître plus clair, plus juste et plus complet, en d'autres mots, d'acquérir une réputation de fiabilité plus grande.

\section{D'UNE ÉDITION À L'AUTRE...}

2.1. ROBERT \& COLLINS : ANALYSE DES TROIS ÉDITIONS 1978, 1987, 1993

\subsubsection{Présentation générale}

Comptant dix-sept années d'existence, le Robert \& Collins jouit d'une bonne réputation et constitue l'objet de référence de la majorité des étudiants. De ce fait, il m'a paru intéressant d'analyser le traitement qui est fait des différents niveaux de langue correspondant à ce qu'il est convenu d'appeler non-standard, et ce, à travers les trois éditions existantes : 1978, 1987 et 1993.

Se targuant, dès la première page d'introduction, de «s'attacher à présenter au lecteur une image fidèle de la langue telle qu'elle est parlée quotidiennement, lue dans les journaux et les revues, parlée en société, entendue dans la rue», nous nous proposons de voir dans quelle mesure le traitement lexicographique a évolué dans le souci de toujours refléter aussi fidèlement la réalité linguistique de la rue.

L'introduction ne varie guère d'une édition à l'autre. Il faut toutefois noter un argument d'importance davantage développé dans la dernière édition, qui concerne l'étendue géographique du corpus. Si les éditions de 1978 et 1987 prennent en compte, outre l'anglais de Grande-Bretagne et le français de France, les termes courants de l'anglais d'Amérique et du français du Canada, l'édition de 1993 met l'accent sur l'anglais nord-américain et introduit les termes les plus répandus de Belgique et de Suisse. Cette modification est de taille pour le sujet qui nous concerne, puisque le lexique utilisé outreAtlantique constitue un puits d'innovations pour la langue non standard.

Sans revenir sur les critiques qui ont été faites au paragraphe 1.1.1., il est nécessaire de faire remarquer les changements opérés dans les exemples cités dans la partie réservée 
aux conseils d'utilisation de l'ouvrage. Si, pour illustrer les trois niveaux de langue notés *, ** et $* * *$, les deux premières editions utilisaient respectivement fra. charabia, fra. c'est du gâteau; fra. se faire pigeonner et fra. baiser, la nouvelle édition cite fra. écraseur, fra. discuter du sexe des anges; fra. doucement les basses, et garde fra. baiser, les lexies remplacées gardant la même étiquette dans le corpus ${ }^{6}$. Ceci est la preuve d'un souci d'innovation, même si le choix d'écraseur est contestable dans le sens où il est très rarement utilisé et ne reflète donc pas justement la réalité langagière.

L'édition 1993 bénéficie d'une légère modification mais qui peut néanmoins être qualifiée d'heureuse puisque les astérisques et leur signification apparaissent à la fin de la liste des abréviations, évitant ainsi au lecteur de se reporter à une page ultérieure.

\subsubsection{Quelques articles types}

Dans le corps même du dictionnaire, c'est-à-dire les mots adresse et leurs articles, les remaniements sont de plusieurs types, allant d'une variation à un réel changement, comme en témoignent les exemples suivants.

Ajout d'une précision

(1978) ang. scab: $\left({ }^{* *} \text { pej : Ind }\right)^{7}$ jaune*

(1987 \&1993) scab: (** pej: strikebreaker) jaune*

L'ajout de cette précision facilite la compréhension aux non-initiés à cette pratique.

- Modification de la définition

(1978) ang. scary* : qui met mal à l'aise, qui donne des frissons

(1987) scary* : qui donne des frissons or la frousse*, angoissant

(1993) scary* : qui donne des frissons or la frousse*, effrayant, angoissant

La modification apportée dans l'édition de 1987 a l'avantage de donner un équivalent appartenant au même niveau de langue.

- Suppression d'un équivalent sémantique dans la définition et ajout d'un autre

(1978) ang. sap**: (fool) cruche*, gogo*

(1987 \& 1993) sap** : (fool) cruche*, andouille*

(1978) fra. il a gagné les doigts dans le nez* : he romped home

(1987 \& 1993) il a gagné les doigts dans le nez ${ }^{*}:$ he won hands down*

Le remplacement de gogo par andouille illustre la volonté des auteurs de se mettre au goût du jour et évite ainsi au lecteur un «anachronisme».

- Suppression d'un astérisque

(1978) ang. sacred cow* : chose sacro-sainte

(1987 \& 1993) sacred cow: chose sacro-sainte

(1978) ang. sales talk* : boniment (often pej) baratin**

(1987\& 1993) sales talk* : baratin publicitaire, boniment

La suppression d'un astérisque peut concerner plusieurs niveaux de langue et affecte soit le mot adresse, soit l'équivalent donné dans l'autre langue.

- Ajout d'un ou plusieurs astérisque(s)

(1978) ang. darkey**, darkie**: (pej) moricaud, nègre (pej) négresse

(1987) darkey**, darkie ${ }^{* *}:$ (pej) moricaud, nègre (pej) négresse

(1993) darkey***, darkie ${ }^{* * *}:$ (pej) moricaud ${ }^{* * *}$, nègre ${ }^{* * *}$ (pej) négresse ${ }^{* * * *}$

(1978 \& 1987) fra. il $y$ en a pagaie / pagaille: there are loads* ou masses of them

(1993) il y en a pagaie / pagaille* : there are loads* ou masses of them 
L'absence d'astérisques dans la définition de darkey des deux premières éditions est quelque peu surprenante. Le lecteur a dû en déduire que les équivalents français n'étaient en rien choquants ou insultants ! On peut se demander si l'ajout d'un astérisque aux deux mots adresse répond au débat sur la «political correctness»!

- Entrée d'un sens non standard étasunien

(1978) ang. scandalmonger: mauvaise langue, colporteur (-euse) de ragots* (1987 \& 1993) scandalmonger: mauvaise langue, colporteur (-euse) de ragots*; (US press) : torchon*

- Entrée d'un sens non standard

(1978 \& 1987) le sens non standard n'apparaît pas (1993) ang. scalp: $\left({ }^{*}\right.$ St Ex) boursicoter

(1978 \& 1987) le sens non standard n'apparaît pas (1993) fra. cageot ${ }^{* *}$ (femme laide) dog**

- Suppression d'un sens non standard et ajout du signe + : emploi vieilli

(1978) ang. funk* : froussard(e)* trouillard(e)** to be in a blue funk avoir la frousse* or la trouille**

(1987) funk* : (Brit) to be in a (blue) funk (frightened) avoir la trouille**

(1993) funk* (Brit + ) to be in a (blue) funk (frightened) avoir la trouille**

- Entrée d'une lexie composée à l'intérieur d'un article

(1978) la lexie composée n'apparaît pas

(1987 \& 1993) ang. Saturday night special* : révolver bon marché

- Entrée d'une lexie complexe

(1978) la lexie complexe n'apparaît pas

(1987 \& 1993) ang. back to the salt mines $!^{*}:$ allez, il faut reprendre le collier !*

(1978) la lexie complexe n'apparaît pas

(1987 \& 1993) fra. avoir du pain sur la planche* : to have a lot to do

- Entrée d'un mot adresse

(1978 \& 1987) le mot n'apparaît pas

(1993) ang. sarnie :* (Brit) sandwich, casse-dallc ${ }^{8}$

(1978) le mot n'apparaît pas

(1987 \& 1993) fra. pacson ** : packet

Il s'agit bien entendu des types les plus récurrents de modification qui peuvent se combiner entre eux.

\subsubsection{Peut-on parler de réelle évolution?}

Les changements qui viennent d'être mentionnés témoignent d'une évolution d'une édition à l'autre, ne serait-ce que dans la volonté des auteurs d'élargir leur champ d'investigation et dans leur souci de faciliter la consultation de l'ouvrage. Plus sérieusement, les modifications concernant le traitement des lexies non standard sont-elles à considérer en terme d'amélioration?

Les types fréquents de modification prouvent que les ajouts, qui concernent les mots adresse, les sens et les astérisques, sont beaucoup plus nombreux que les retraits ou suppressions qui portent le plus souvent sur des parties de définition. Cela revient à dire que les auteurs ont davantage tenu compte des néologismes non standard et ignoré la disparition d'usage de certaines lexies et de certains sens. On peut en effet se réjouir de l'apparition dans l'édition 1993 de lexies telles que ang. glitterati*: «le beau monde, les rupins*»; ang. headbanger*: «un(e) enragé(e)* de heavy metal, un(e) cinglée*»; fra. 
caf(e)teur*/caf(e)teuse, fra. pactole* et déplorer l'absence de ang. grody**: «vil, révoltant, minable», par exemple, en usage depuis un certain nombre d'années ${ }^{9}$. De même, il peut paraître surprenant de trouver des mots tels que fra. jactance**: «chat»; fra. jobarderie* : «gullibility» ou fra. ragougnasse* : «pigswill» dans I'édition 1993 non accompagnés du signe + marquant l'archaïsme. On ne peut considérer l'espérance de vie des mots non standard de la même manière que celle du lexique standard.

Dans le même ordre d'idée, un mot qui dans la première édition est accompagné d'un ou de plusieurs astérisques, signe de son appartenance au lexique non standard, peut, au fil du temps et au gré des modes, changer de registre jusqu'à faire partie intégrante de la langue non standard. C'est le cas de savvy: «jugeotte, bon sens», qui est noté ** dans l'édition de 1978 puis * dans les éditions de 1987 et 1993. Ainsi, 1'appartenance d'une lexie à un registre n'est jamais fixée définitivement, elle est en perpétuelle évolution. Même s'il est vrai qu'un mot est davantage susceptible de perdre un astérisque et, par là même, d'approcher du standard, il est des cas, beaucoup plus rares, pour lesquels la progression se fait en sens inverse ${ }^{10}$.

\subsection{HARRAP'S SLANG DICTIONARY : ANALYSE DES DEUX ÉDITIONS : 1984 ET 1993}

\subsubsection{Présentation générale}

Dix années environ séparent la première (1984) de la deuxième édition (1993) du Harrap's Slang Dictionary, dix années, qui, si l'on en croit la première phrase de la préface de la seconde édition, ont été marquées par une évolution de la langue non standard. Nous nous proposons donc de vérifier si l'édition 1993 reflète fidèlement cette progression dans les changements qui ont été opérés.

La préface présente quelques modifications bienvenues pour la plupart. Le nombre d'étiquettes utilisées pour faire référence au non-standard baisse sensiblement, ce qui a pour avantage de réduire l'impression de manque de rigueur et de sérieux que pouvait ressentir le lecteur. Ce qui était nommé «expressions familières et argot communément employés» a heureusement disparu. En effet, comment l'argot, qui est par définition une langue cryptique, peut-il être communément employé sans perdre par là même sa caractéristique première? Il faut noter également que la préface de l'édition 1993 est un peu plus prolixe quant aux sources utilisées, la préface 1984 ne les mentionnant à aucun moment.

Paradoxalement, l'édition 1993 voit le nombre d'abréviations correspondant aux différents registres augmenter. La mention Sl (slang; expression argotique) fait son entrée aux côtés de $F, P, V$ et $V V$, ce qui n'est pas sans poser des problèmes sur lesquels nous ne reviendrons pas ici ${ }^{11}$. Enfin, la seconde édition laisse présager des changements puisque l'accent est mis sur le caractère éphémère du non-standard, cela étant dô, entre autres, à l'influence de la mode et à l'évolution des tabous.

\subsubsection{Quelques articles types \\ Certaines modifications ont été opérées dans le corpus : nous allons citer ici les plus importantes.}

Nouvelle entrée

(1993) fra. à l'aise $\mathrm{F}$ : easily

fra. faire ami-ami $\mathrm{F}$ : to make friends; (se réconcilier) to make up

fra. café-couette F : (en Bretagne) bed and breakfast/b. \& b. ${ }^{12}$

fra. on ne tire pas sur une ambulance $\mathrm{F}$ : you don't hit a man who's down ang. dank NAm P : marijuana, herbe ang. ofay NAm P Pej: (white person) Blanc, Blanche 
Suppression d'un mot adresse, d'une expression

(1984) fra. l'avoir amère $F$ : to be indignant/annoyed

fra. cadière $\mathrm{F}$ : chair

ang. to dab one's nose $\mathrm{F}$ : se poudrer le nez, se refaire une petite beauté

- Suppression d'un mot adresse noté comme archaïque ou vieilli

(1984) fra. agrafe A P : hand/(meat) hook

fra. allezingue A P : jacket

fra. caire OP: money/ackers/shekels

ang. dab down O P (régional) donner de l'argent, abouler son fric

- Suppression d'un équivalent dans la définition

(1984) ang. dabble F: se droguer irrégulièrement, (s)chnoufailler

(1993) dabble $\mathrm{F}:$ se droguer irrégulièrement

\subsubsection{Peut-on parler de réelle évolution?}

La liste précédente fait état d'un certain nombre de modifications qui se matérialisent le plus souvent par des entrées et des sorties de mots adresse. Les nouveautés sont en nombre important, même si elles ne sont pas toutes judicieuses dans le sens où elles ne sont plus utilisées; c'est le cas par exemple de fra. cageot: «unattractive/ugly woman or girl» ou de fra. avoir l' oil américain: «to be able to judge (sthg) accurately at a single glance». Les suppressions de mots adresse ne concernent pas uniquement les mots dont l'usage était noté comme vieilli ou archaïque dans l'édition 1984, comme en témoignent les exemples suivants : fra. cafarde $\mathrm{P}$ : «moon», fra. allumettes $\mathrm{F}$ : «long skinny legs / matchsticks» ou ang. oil-can NAm P: «échec, four fiasco». Si cafarde peut en effet être considéré comme un archaïsme, il semble que allumettes soit toujours utilisé. Faut-il en déduire que ce mot est devenu standard, ce dont nous doutons fortement, ou qu'il s'agit d'un oubli des auteurs?

Pour ce qui est des différentes étiquettes correspondant aux registres, les modifications sont minimes alors que certains mots auraient été susceptibles de passer de $\mathrm{P}$ à $\mathrm{F}$, comme par exemple: fra. fiole: «head»; fra. flemme: «laziness»; fra. Mondaine: «the vice squad» ou encore fra. capote anglaise: «condom»13. De la même manière, des mots tels que fra. forcir: «to get fat»; fra. coup de foudre: «love at first sight»; fra. moto: «motorbike»; fra. ribambelle: «crowd» méritent-ils d'apparaître dans la deuxième édition du Harrap's Slang Dictionary alors qu'ils semblent considérés comme standard depuis un certain nombre d'années?

$\mathrm{Si}$, effectivement, il y a eu une évolution entre les deux éditions du Harrap's Slang Dictionary, ce qui vient d'être dit prouve que les différents mots n'ont pas subi le même traitement ou la même attention de la part des auteurs. L'accent a été mis sur les nouveautés au détriment du corpus déjà existant qui aurait mérité davantage de vigilance.

\section{QUELLES SOLUTIONS ?}

L'état des lieux, suivi de l'analyse de l'évolution de deux dictionnaires d'une édition à l'autre font apparaître un certain nombre de problèmes de nature différente, relatifs, d'une part à la notion de non-standard et, d'autre part, à l'ouvrage dictionnairique. Entreprendre un travail dont l'objet est la langue non standard, c'est accepter de traiter cette partie de la langue que tout le monde est à même de reconnaître mais que personne n'est apte à définir de manière satisfaisante pour des raisons qui lui sont inhérentes. Sans faire état ici de toutes ses propriétés, il est essentiel de citer celles qui ont une influence directe sur le travail lexicographique. Ephémère, elle change au fil du temps et au gré des modes; subjective, elle a une dimension esthétique (beau/laid), morale (bien/mal) et hygiénique 
(propre/sale), aspects qui sont liés de très près à la culture dans laquelle elle évolue. Cela ne signifie pas pour autant que le traitement qui en est fait se doit d'être vague et approximatif. Trop souvent, l'analyse des dictionnaires bilingues fait ressortir des lacunes identiques qui peuvent, pour la plupart, être comblées.

\subsection{NIVEAUX DE LANGUE}

Les registres ou niveaux de langue constituent sans aucun doute le problème central. Le fait qu'étant subjectives dans la réalité, les limites qui les séparent sont floues et fluctuantes ne doit pas servir d'excuse pour un traitement anarchique à l'intérieur d'un même dictionnaire, ni pour une multitude d'étiquettes d'un ouvrage à l'autre. Une certaine rigueur est nécessaire pour pallier ce manque de précision. L'éventail est en effet déroutant: certains utilisent une échelle graduée de quatre niveaux de langue, d'autres de trois. D'un côté sont recensées les étiquettes familier (familiar, colloquial); populaire, argotique (popular, slang); trivial (vulgar); obscène, ordurier (very vulgar, taboo); d'un autre argot (slang); familier (informal); très familier (very informal) et vulgaire (offensive); puis familier (informal); populaire (very informal); vulgaire ou tabou (vulgar or taboo); et enfin argot (slang); familier (familiar, colloquial); vulgaire (vulgar). Plus déconcertant encore est le fait que l'étiquette argot corresponde tantôt à ce que certains appellent populaire, d'autres très familier, tantôt à ce qui est considéré comme langue cryptique d'un groupe social, sans mentionner que dans les deux cas, on lui donne l'équivalent anglais de slang. Cet état de fait est à l'origine de différences d'un dictionnaire à l'autre dans les registres qui sont conférés aux adresses et à certains de leurs sens. Le tableau qui suit en est une illustration parlante.

\begin{tabular}{|l|c|c|c|c|}
\hline Mot ou expression & $\begin{array}{c}\text { Robert \& Collins } \\
1993\end{array}$ & $\begin{array}{c}\text { Hachette-Oxford } \\
1994\end{array}$ & $\begin{array}{c}\text { Harrap's Shorter } \\
1993\end{array}$ & $\begin{array}{c}\text { Harrap's Slang } \\
1993\end{array}$ \\
\hline miel ! (sugar!) & $*$ & sens absent & $\mathrm{F}$ & $\mathrm{P}$ \\
mimi (little kiss) & $+^{*}$ & non étiqueté & non étiqueté & vieilli F \\
revouloir (to want again) & $*$ & mot absent & $\mathrm{F}$ & $\mathrm{F}$ \\
ricain (Yankee) & $* *$ & 0 & sens absent & $\mathrm{P}$ \\
terrine (head) & $* *$ & sens absent & $\mathrm{F}$ & $\mathrm{F}$ \\
\hline binge (beuverie) & $* *$ & 0 & mot absent & $\mathrm{P}$ \\
bint (nana) & $* *$ & 0 & mot absent & mot absent \\
biopic (film biographique) & $*$ & 0 & sens absent & sens absent \\
eggbeater (hélico) & $* *$ & 0 & $\mathrm{~F}$ & $\mathrm{~F}$ \\
pep (entrain) & $*$ & non étiqueté & & \\
\hline
\end{tabular}

S'agissant d'ouvrages dont les dates d'édition sont sensiblement identiques, les différences sont importantes, a fortiori lorsqu'il s'agit de deux dictionnaires, l'un général, l'autre spécialisé, issus de la même maison d'édition... Il semble nécessaire ici de procéder à un changement radical dans la manière de présenter ce qui a trait au non-standard, ce qui présuppose un certain nombre d'étapes à suivre. Il est du devoir des lexicographes de présenter clairement dans l'introduction ou dans les conseils d'utilisation la manière dont ils l'appréhendent. En d'autres mots, ils doivent énumérer et définir les registres qu'ils utilisent, aussi précisément que cela est possible, sans omettre de spécifier les critères de sélection qu'ils ont choisis. Dans un deuxième temps, le code sélectionné doit être 
précisé, suivi d'avertissements prodigués au lecteur sur les conditions d'usage de chacun et les risques encourus. L'ajout d'une abréviation «Irr» pour irrespectueux pourrait peutêtre limiter les pièges stylistiques tendus au locuteur étranger. Quoi qu'il en soit, et ce détail pratique est d'importance, il semble que ce soit le type de code utilisé par le Robert $\&$ Collins (système d'astérisques) et par le dictionnaire Hachette-Oxford (système de ronds) qui soit le mieux adapté, étant plus facilement repérable dans le texte que ne l'est le système de lettres choisi par Harrap's.

\section{2. ÉQUIVALENTS TENANT LIEU DE DÉFINITION}

Les équivalents dans l'autre langue donnés aux adresses et à leurs différents sens n'est pas non plus sans poser de problèmes, même s'il est vrai que la différence de lexicalisation des concepts est moins marquée dans des langues voisines telles que l'anglais et le français. Très souvent, les unités lexicales ont la même dénotation mais ne sont pas des synonymes absolus, leur connotation et les niveaux de langue auxquels ils appartiennent étant différents, les collocations dans lesquelles elles entrent n'étant pas non plus comparables. Sans qu'il soit question de chercher l'impossible, c'est-à-dire la synonymie parfaite, il est nécessaire de faire un effort pour trouver l'équivalent le plus adéquat: il devra avoir le même contenu conceptuel et un halo associatif quasiment identique. Pour ce qui est de l'appartenance à un même niveau de langue, la tâche est plus ardue, les ressources des deux langues n'étant pas illimitées. Il est nécessaire par conséquent de donner un équivalent standard et un équivalent non standard en prenant soin d'en préciser le registre.

\subsection{NÉOLOGISMES ET ARCHAISMES}

La langue non standard, et il s'agit là d'une caractéristique première énoncée à plusieurs reprises, est sujette à une évolution rapide. Très souvent, dans la préface des dictionnaires, la volonté des auteurs de la représenter fidèlement est mise en avant. Cependant dans la réalité, l'effort que demande la mise à jour permanente de la langue non standard ne semble pas pleinement produit. Même si aucun dictionnaire ne peut prétendre à la complétude et à la perfection, il est des omissions et des inadéquations qui ne sont guère pardonnables. Il est vrai que le lexicographe ne peut recueillir toutes les innovations faites chaque jour dans la rue (c'est là le caractère essentiel de la création non standard), la règle voulant que seuls les termes attestés à l'écrit soient pris en compte. Cependant, comme dans la mode à laquelle le non-standard est soumis, il existe des incontournables qui devraient avoir droit de cité. De la même manière que des mots ou des emplois entrent dans la langue, d'autres sont abandonnés définitivement ou temporairement, et il est essentiel que les lexicographes signalent leur caractère désuet. Trop souvent, de nouvelles entrées sont faites dans le dictionnaire alors qu'elles ne sont déjà plus utilisées. Dans ce domaine également, les différences d'appréciation varient d'un ouvrage à un autre, ce qui est regrettable. Les mouvements de va-et-vient qui animent la langue non standard nécessitent une remise à jour fréquente et systématique qui pourrait être viable à condition que les éditions soient renouvelées ou, faute de mieux, révisées plus souvent. La fluidité du non-standard et la rigueur dictionnairique sont difficilement compatibles...

\subsection{DE LA TRANSPARENCE AVANT TOUTE CHOSE}

De manière plus générale, la plupart des dictionnaires pêchent par un manque d'informations destinées au lecteur relatives à leur manière de procéder. Sans revenir sur ce qui a été dit dans les paragraphes précédents, il serait judicieux de préciser les sources utilisées, les variétés géographiques prises en compte, et de développer les différentes étapes qui constituent le travail lexicographique. Le lecteur ne manquera pas d'apprécier l'innovation faite en ce domaine par le dictionnaire Hachette-Oxford qui joue la carte de la transparence. Espérons qu'il s'agit là d'une tendance qui fera des adeptes. 
L'examen de l'activité dictionnairique face au non-standard fait ressortir un certain nombre de points à reconsidérer afin d'améliorer la situation. L'incompatibilité déjà mentionnée entre les deux ne peut totalement excuser le caractère incomplet et aléatoire du traitement lexicographique, même si aucun dictionnaire ne peut prétendre à l'exhaustivité et à la perfection. Il est en effet utopique d'imaginer un ouvrage dictionnairique de taille maniable répertoriant toutes les innovations, donnant toutes les nuances sémantiques d'un mot. Cependant, la distinction s'impose ici entre dictionnaires généraux et dictionnaires spécialisés. Si l'on admet plus facilement les omissions et inadéquations dans les premiers, on a plus de mal à les pardonner dans les deuxièmes, vis-à-vis desquels on est logiquement plus exigeant.

Il semble qu'à ce point, le traitement dictionnairique du non-standard exige un manquement à la règle lexicographique qui prévaut dans le recueil même des mots. Le domaine de prédilection de la création lexicale non standard est majoritairement l'oral, et il est nécessaire d'en tenir compte si l'on veut présenter une image fidèle de la langue. Le fait de se limiter aux termes attestés à l'écrit a pour conséquence majeure de présenter un lexique déjà démodé au moment de la parution du dictionnaire. Cela se matérialise entre autres par l'apparition de lexies qui ne sont plus en usage, par la présence d'unités lexicales qui ne sont pas notées archaïque, et par une attribution inadéquate des niveaux de langue.

Concernant ce dernier point, on se demande s'il ne serait pas utile de se livrer à une étude contrastive portant sur l'étiquetage des différents mots et sens en anglais et en français. Cette analyse comparative aurait un double but: elle permettrait peut-être de voir si les niveaux de langue conférés peuvent être fonction de champs notionnels spécifiques, et dans quelle mesure les différences culturelles et surtout les tabous influencent notre vision de la langue.

\section{Notes}

* Cet article est issu d'une communication présentée par l'auteur aux IVes Journées scientifiques du réseau «Lexicologie, terminologie, traduction» de l'AUPELF-UREF (Lyon, France, 28, 29, 30 septembre 1995).

1. Robert \& Collins, 1993 : xiv

2. Robert \& Collins, 1993 : xxvii, xxviii.

3. Hachette-Oxford, 1994, préface.

4. Mot qui trouve son origine dans la musique rap des États-Unis.

5. Harrap's Slang Dictionary, 1993 : viii.

6. Les équivalents anglais étant to make a bolt for it: *filer* ou se sauver à toutes jambes, he's pretty hot* at football: il est très calé en foot*; bigwig* grosse légume**, huile**; you bloody fool !***; espèce de con !*** qui ont cédé la place à kidology*: bluff, he laughed himself silly*: il a ri comme un bossu* ou comme une baleine* kisser $^{* *}$ : gueule ${ }^{* *}$ et fart f*** $^{* *}$ : péter***.

7. J'ai dû en vérifier le sens dans le dictionnaire, la définition étant insuffisante.

8. Il est surprenant que ce composé ne soit pas noté *.

9. Cette lexie est en usage depuis les années 60 , et a été très à la mode dans les années 80 .

10. Cf. darkey / darkie mentionné en 2.1.2.

11. Cf. 1.2.1.

12. Il semble que ce mot soit également employé dans d'autres régions françaises !

13. Ces mots sont notés comme familiers dans Le Petit Robert, édition 1984 !

\section{RÉFÉRENCES}

Harrap's Shorter French and English Dictionary (1991): 2nd ed., reprinted with supplement 1993, 1994, 1995 , London, Harrap.

Harrap's Slang French-English Dictionary $(1984,1993): 1$ st and 2nd ed., London, Harrap.

Le Dictionnaire Hachette-Oxford français-anglais / anglais-français (1994) : Paris, Oxford, Hachette-Oxford.

Oxford Dictionary of New Words (1991): Oxford, Oxford University Press.

Robert \& Collins Senior: Dictionnaire français-anglais, anglais-français $(1978,1987): 1^{\mathrm{re}}, 2^{\circ}$ et $3^{\circ}$ éditions, Paris, Londres, Robert-Collins. 